\title{
Clinical Utility of the Korean Version of the WHO Adult Attention- Deficit/Hyperactivity Disorder Self-Report Scale Screener
}

\author{
Simyang Heo ${ }^{1}$, Ji-Hae Kim ${ }^{1}$, Yoo-Sook Joung ${ }^{1}$, Won-Ik Lee ${ }^{2}$, \\ Joo-Jin $\mathrm{Kim}^{3}$, Seok Han Sohn ${ }^{4}$, and Soon Ah Chang ${ }^{5}$ \\ 1'Department of Psychiatry, Samsung Medical Center, Sungkyunkwan University, School of Medicine, Seoul, Republic of Korea \\ ${ }^{2}$ Maumnuri Clinic, Seoul, Republic of Korea \\ ${ }^{3}$ Yuil Medical Clinic, Guri, Republic of Korea \\ ${ }^{4}$ Yonsei Psychiatric Clinic, Seoul, Republic of Korea \\ ${ }^{5}$ Cheongdam Yonsei Premier Clinic, Seoul, Republic of Korea
}

\begin{abstract}
Objective The aim of this study was to compare the performance of the 18-item Korean version of the World Health Organization adult attention-deficit/hyperactivity disorder self-report scale (ASRS) with the six-item ASRS Screener for predicting attention-deficit/ hyperactivity disorder (ADHD) group.

Methods The study sample included 51 adult patients with ADHD and 158 normal controls. All participants completed the ASRS and were interviewed individually using the Mini-International Neuropsychiatric Interview. Receiver operating characteristic (ROC) curves were used to compare the ASRS (ASRS-18) with the ASRS Screener (ASRS-6) in Korean samples.

Results The ADHD group had higher ASRS and ASRS subscale scores than those of the control group. ROC curve analysis revealed the ASRS was more powerful to predict ADHD group than the ASRS Screener, but the ASRS Screener also had strong concordance with clinician diagnoses.

Conclusion This study shows that the 18-question ASRS outperforms the six-question ASRS Screener. However, the weighted Screener is also a valid and useful screening instrument both in epidemiological surveys and in clinical settings.
\end{abstract}

Psychiatry Investig 2018;15(3):325-329

Key Words Adult ADHD, ASRS, ARSR screener, ROC.

\section{INTRODUCTION}

Although it has long been known that attention-deficit/hyperactivity disorder (ADHD) remits in children, $\mathrm{ADHD}$ may persist into adulthood in a large proportion of cases. ${ }^{1,2}$ Surveys of $18-44$-year-old adults show that $4.4 \%$ meet the crite-

Received: February 16, 2017 Revised: June 13, 2017

Accepted: July 10, 2017

$\triangle$ Correspondence: Ji-Hae Kim, PhD

Department of Psychiatry, Samsung Medical Center, Sungkyunkwan University School of Medicine, 81 Irwon-ro, Gangnam-gu, Seoul 06351, Republic of Korea Tel: +82-2-3410-0931, Fax: +82-2-3410-0050

E-mail: jihae0931.kim@samsung.com

$\triangle$ Correspondence: Yoo-Sook Joung, MD, PhD

Department of Psychiatry, Samsung Medical Center, Sungkyunkwan University School of Medicine, 81 Irwon-ro, Gangnam-gu, Seoul 06351, Republic of Korea Tel: +82-2-3410-0930, Fax: +82-2-3410-0050

E-mail: yschoung.joung@samsung.com

(a) This is an Open Access article distributed under the terms of the Creative Commons Attribution Non-Commercial License (http://creativecommons.org/licenses/bync/4.0) which permits unrestricted non-commercial use, distribution, and reproduction in any medium, provided the original work is properly cited. ria for the $\mathrm{ADHD}$ diagnosis 2, and 3-6\% of adults have $\mathrm{AD}$ HD-like symptoms that interfere with daily life. ${ }^{3}$

The core symptoms of ADHD are hyperactivity, impulsivity, and inattention. Untreated ADHD results in underachievement, failure in academic activities, and dropping out of academic frameworks. In addition, adult employees with ADHD have lower levels of work performance, task completion, and poorer relationships with supervisors. ${ }^{4}$ This poor work performance can lead to higher rates of unemployment, frequent job changes, and lower socioeconomic status. ${ }^{5}$

Despite the prevalence of ADHD in adults, it seems to be underdiagnosed or misdiagnosed. The rate of treatment of ADHD in adults is much lower than its prevalence ${ }^{6}$; only $11 \%$ of respondents in a 2006 survey who met the criteria for adult ADHD had been treated in the previous 12 months. ${ }^{2}$ The reason for the underdiagnosis of adult ADHD is probably due to the time-consuming assessment, which requires specific knowledge and a specialist. Furthermore, many tests 
that evaluate attention take longer than 90 minutes to complete, and include clinical interviews and rating scales. ${ }^{7}$

Several Adult ADHD screening tests have been developed, including the Connors' Adult ADHD Rating Scale, ${ }^{8}$ the Brown Attention Activation Disorder Scale, ${ }^{9}$ the Wender Utah Rating Scale, ${ }^{10}$ and the Adult ADHD Self-Report Scale. ${ }^{11}$ These selfassessments were originally intended for clinical use, but they have been used extensively in research. They all show good screening quality compared to diagnostic interviews in a clinical setting.

Although a number of measures exist to screen for adult ADHD, ${ }^{12}$ the World Health Organization (WHO) Adult Attention-Deficit/Hyperactivity Disorder Self-Report Scale (ASRS) used in a U.S. national survey appears to be the most promising scale. ${ }^{13}$ The ASRS includes 18 questions regarding the frequency of recent DSM-IV Criterion A symptoms of adult ADHD and may offer several advantages over existing scales, as it provides a context basis for assessing $\mathrm{ADHD}$ symptoms and can be used to longitudinally examine ADHD symptoms from adolescence to adulthood.

Kessler et al. ${ }^{13}$ developed a short-form ASRS Screener (six questions) to maximize clinical utility. Stepwise logistic regression was used for this evaluation, beginning with selecting the dichotomously coded screening questions that most accurately predicted the clinical syndrome classifications. The ASRS Screener has adequate sensitivity and specificity in the general U.S. population. ${ }^{13}$ However, the only U.S. ASRS validation study was carried out in conjunction with a nationally representative U.S. survey.

The aim of the present study was to compare the performance of the 18-item Korean version of the WHO ASRS with the sixitem ASRS Screener for predicting ADHD group in Korean samples. This study concomitantly presented the cutoff scores separating adult patients with ADHD and normal controls for the ASRS and ARSR Screener.

\section{METHODS}

\section{Participants}

The study population included 51 adult patients with ADHD and 158 normal controls. Adult patients with ADHD were recruited from psychiatric outpatients attending the Samsung Medical Center, Seoul, Korea. Master's-degree level clinical psychologists had diagnosed adult ADHD using the DSMIV and the Mini-International Neuropsychiatric Interview (MINI). ${ }^{14}$ MINI focuses on the existence of current disorders in order to keep it short. Using only one or two screening questions, clinical psychologists could rule out the diagnosis when participants answered negatively. All patients had received a diagnosis of ADHD by use of the MINI. And comorbidity with other psychiatric disorders was included, as long as the criteria for ADHD was fulfilled before the comorbid disorder appeared. Participants who were unable to reliably report ADHD symptoms as determined by researchers were excluded. Also, individuals were excluded when having serious psychotic disorders. Our controls were solicited via advertisements requesting volunteers for various psychological assessments in universities of Kyoung-Ki District and the Seoul Metropolitan area, Korea. The mean age of all subjects was 23.64 years [standard deviation $(\mathrm{SD})=5.91$; range, $18-45$ years]. Written informed consent was obtained from all participants. This study was approved by the Institutional Review Board of the Samsung Medical Center (2009-07-042).

\section{Measures}

\section{Adult ADHD self-report scale}

The ASRS is an 18-item self-report scale, based on the DSMIV symptom list, developed by the Workgroup on Adult ADHD in conjunction with the WHO. ${ }^{13}$ The scale focuses on symptom frequency rather than severity to make scale instructions easier for participants to understand. ${ }^{15}$ Each ASRS question asks respondents how often a particular ADHD symptom has occurred over the past 6 months using a 5-point response scale ranging from never 0 ), rarely 1 ), sometimes 2 ), often 3 ), to very often 4). Total scores are 0-72. The Korean version of ASRS has been validated by Kim et al. and displays good validity and reliability. ${ }^{16}$

\section{ASRS screener}

The ASRS Screener, which is the short form of the ASRS, consists of 6 of the 18 ASRS questions selected based on stepwise logistic regression, beginning with selection of the dichotomously coded screening questions that most accurately predicted the clinical syndrome. ${ }^{13}$ The first four questions address inattention and the last two questions addressing hyperactivity. ${ }^{17}$ The ASRS Screener score is the sum of the dichotomous responses for the six questions. Kessler et al. ${ }^{13}$ identified thresholds for each item with maximum concordance with interview results.

\section{MINI PLUS 5.0 structured interview}

The MINI is a short structured diagnostic interview developed jointly by psychiatrists and clinicians in the U.S. and Europe for DSM-IV and ICD-10 psychiatric disorders. It is applied to assess lifetime and current DSM-IV Axis I psychiatric morbidities.

\section{Procedure}

The Master's level psychologists interviewed each partici- 
pant using the DSM-IV and the MINI. Participants also completed the ASRS.

\section{Data analysis}

The clinical data and the distribution of scores were analyzed using descriptive methods and the $\chi^{2}$ and $t$ tests were performed using SPSS ver. 17 software (SPSS Inc., Chicago, IL, USA). We used receiver operating characteristic (ROC) curve to examine the performance of the ASRS and the ASRS Screener in relation to ADHD diagnosis. The performance of the two instruments was indicated by the area under the ROC curve (AUC), which describes the relationship between sensitivity and specificity.

\section{RESULTS}

\section{Demographic characteristics}

The demographic characteristics of the ADHD and control groups are described in Table 1 . The mean ages of the ADHD and control groups were 27.82 years $(\mathrm{SD}=8.89$; range, $18-45$ years) and 22.29 years ( $\mathrm{SD}=3.68$; range, $18-37$ years), respectively $(\mathrm{t}=-4.322, \mathrm{p}<0.001)$. Additionally, significant differences in sex distribution $\left(\chi^{2}=8.598, \mathrm{df}=1, \mathrm{p}=0.003\right)$ and education level $\left(\chi^{2}=22.749, \mathrm{df}=3, \mathrm{p}<0.001\right)$ were detected between the groups.

\section{Differences in the ASRS and the ASRS screener between the groups}

The validity of the ASRS was assessed by comparing the scores of the participants with and without an independent clinician's diagnosis of ADHD. Group differences were tested with the t-test. The mean total ASRS score was 37.65 (SD=11.24) for the $\mathrm{ADHD}$ group and 24.99 ( $\mathrm{SD}=8.59)$ in the control group $(\mathrm{p}<0.001)$. Mean ASRS Screener score was significantly different between the two groups (Table 2). All mean total ASRS, ASRS subscale, and ASRS Screener scores of participants with
ADHD were significantly higher than those of the control participants $(\mathrm{p}<0.001)$.

\section{ROC analysis of the ASRS and the ASRS screener}

Figure 1 presents the ROC curves for the total ADHD score and the ADHD Screener score. The area under the curve (AUC) and the 95\% confidence intervals are shown in Table 3 . The ASRS had an AUC of 0.810 for differentiating ADHD correctly from the control, whereas the ASRS Screener had an AUC of 0.751. A large AUC indicates small false-negative and false-positive response rates, indicating high sensitivity and specificity. Table 4 presents the sensitivity and specificity values for the ASRS and ASRS Screener. The ASRS had sensitivity and specificity scores of 0.706 and 0.804 , respectively, when using the optimal cutoff score of 32 . The ASRS Screener showed psychometric properties similar to those of the ASRS, although the sensitivity score (0.627) was lower than in the ASRS, whereas the specificity score (0.804) was similar to the ASRS, when using the optimal cutoff score of 4 for the ASRS Screener.

\section{DISCUSSION}

Similar to other studies, ${ }^{13,17}$ the WHO ASRS and ASRS Screen-

Table 2. Comparison of the ASRS and ASRS Screener scores between the ADHD and control groups

\begin{tabular}{lccccccc}
\hline & $\begin{array}{c}\text { ADHD } \\
(\mathrm{N}=51)\end{array}$ & & $\begin{array}{c}\text { Control } \\
(\mathrm{N}=158)\end{array}$ & $\mathrm{t}$ & $\mathrm{p}$ \\
\cline { 2 - 3 } & Mean \pm SD & & Mean \pm SD & & \\
\hline ASRS total & $37.65 \pm 11.24$ & & $24.99 \pm 8.59$ & -7.371 & $<0.001$ \\
ASRS inattention & $21.39 \pm 5.41$ & & $14.83 \pm 4.74$ & -8.295 & $<0.001$ \\
ASRS hyperactivity & $16.25 \pm 7.07$ & & $10.16 \pm 4.81$ & & \\
ASRS screener & $3.69 \pm 1.50$ & & $2.27 \pm 1.46$ & -5.738 & $<0.001$ \\
\hline
\end{tabular}

ADHD: attention-deficit/hyperactivity disorder, ASRS: Adult ADHD Self-Report Scale, ASRS total (18-item), ASRS Screener: (6-item)

Table 1. Demographic variables of the participants

\begin{tabular}{|c|c|c|c|c|}
\hline & $\operatorname{ADHD}(\mathrm{N}=51)$ & Control $(\mathrm{N}=158)$ & $\mathrm{t}, \chi^{2}$ & $\mathrm{p}$ \\
\hline Age (years) (mean \pm SD) & $27.82 \pm 8.89$ & $22.29 \pm 3.68$ & $\mathrm{t}=-4.322$ & $<0.001$ \\
\hline Gender, N (\%) & & & $\chi^{2}=8.598$ & 0.003 \\
\hline Male & $33(64.7)$ & $65(41.1)$ & & \\
\hline Female & $18(35.3)$ & $93(58.9)$ & & \\
\hline Education, N (\%) & & & $\chi^{2}=22.749$ & $<0.001$ \\
\hline Middle school or less & $2(3.9)$ & $0(0)$ & & \\
\hline High school & $31(60.8)$ & $98(63.2)$ & & \\
\hline College or university & $13(25.5)$ & $57(36.8)$ & & \\
\hline Graduate school & $5(9.8)$ & $0(0)$ & & \\
\hline
\end{tabular}

ADHD: attention-deficit/hyperactivity disorder 


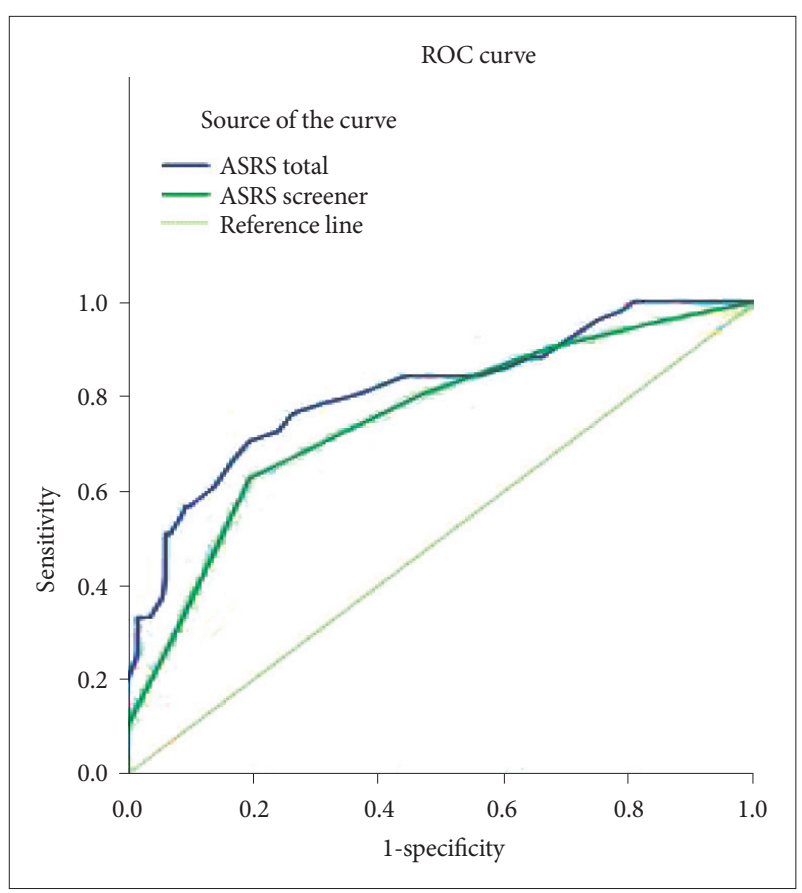

Figure 1. Receiver operating characteristics curves for the study groups. ASRS total, Adult ADHD Self-Report Scale (18-item); ASRS sum 6n, ASRS Screener (6-item). ADHD: attention-deficit/ hyperactivity disorder, ASRS: Adult ADHD Self-Report Scale, ASRS total (18-item), ASRS Screener: (6-item).

Table 3. Receiver operating characteristics analysis for the study groups

\begin{tabular}{lcccc}
\hline & \multirow{2}{*}{ AUC } & p & \multicolumn{2}{c}{$95 \%$ confidence } \\
\cline { 4 - 5 } & & & Low limit & Up limit \\
\hline ASRS total & 0.810 & 0.000 & 0.737 & 0.882 \\
ASRS screener & 0.751 & 0.000 & 0.672 & 0.831 \\
\hline
\end{tabular}

ADHD: attention-deficit/hyperactivity disorder, ASRS: Adult ADHD Self-Report Scale, ASRS total (18-item), ASRS Screener: (6-item)

Table 4. Sensitivity and specificity values of the scales

\begin{tabular}{lccc}
\hline & Sensitivity & Specificity & 1-specificity \\
\hline ASRS total (cutoff=32) & 0.706 & 0.804 & 0.196 \\
ASRS Screener (cutoff=4) & 0.627 & 0.804 & 0.196 \\
\hline
\end{tabular}

ADHD: attention-deficit/hyperactivity disorder, ASRS: Adult ADHD Self-Report Scale, ASRS total (18-item), ASRS Screener: (6-item)

er were effective to evaluate adult ADHD. Participants with ADHD rated themselves higher on the ASRS and the ASRS subscales than those of the normal controls. Results confirmed the Korean version of the ASRS and ASRS Screener as a reliable and valid adult $\mathrm{ADHD}$ screening instrument.

Kessler et al. ${ }^{18}$ found that the ASRS Screener with dichotomized scoring provided the best sensitivity and specificity for diagnosing adult ADHD. The Korean version of the full ASRS outperformed the ASRS Screener. Further research is needed on the ASRS Screener considering of that an Israeli study com- paring the ASRS and the ASRS Screener reported similar results with ours. ${ }^{19}$

However, the ASRS Screener is also a fair test in a high sensitivity and high specificity, and has many utilities. The sixquestion ASRS Screener can be self-administered easily and quickly (less than 2 minutes) both in community surveys and clinical settings. Patients would be willing to administer the screening tool due to its ease to complete brief time. It is also very good tool for trained clinical interviews to conduct clinical evaluations.

Several limitations in this study should be mentioned. First, the majority of our sample was young, which may have overlooked severity and dysfunction of ADHD as well as the range of co-morbid conditions. ${ }^{20,21}$ Second, all data were obtained from respondents rather than from informants. The psychometric properties of a self-report provide lower bound estimates relative to a clinician administered version or an informant's report. ${ }^{20,22,23}$ This is because adults are unaware or insufficiently aware of their own impairments. Third, the sample in this study may have included subjects both on medication and off medication. By not controlling for medication or titration, selection bias may have occurred. Treatment reduces the variability of symptoms in our sample, which may limit generalizability of the results.

The results of the present study indicate that the ASRS and the ASRS Screener are useful. They can be used as general screening tools for patients in a variety of settings due to the high specificity and negative predictive values and identify adults who might require more comprehensive evaluation and follow-up. We also showed that the ASRS was an effective screening tool for adult ADHD. It can also be used as part of an evaluation to obtain data pertaining to several of the ADHD diagnostic criteria. ${ }^{15}$

Overall, it is hoped that the Korean version of the ASRS will be a useful addition to the screening process and aid in correct diagnoses.

\section{Acknowledgements}

The current version of the Adult ADHD Self-Report Scale symptom checklist is copyrighted by the World Health Organization and is available at http://webdoc.nyumc.org/nyumc_d6/files/psych_adhd_checklist.pdf. This project was funded by Janssen Korea Limited (Local Protocol No.: CONKOR-9023).

\section{REFERENCES}

1. Fayyad J, De Graaf R, Kessler R, Alonso J, Angermeyer M, Demyttenaere $\mathrm{K}$, et al. Cross-national prevalence and correlates of adult attention-deficit hyperactivity disorder. Br J Psychiatry 2007;190:402-409.

2. Kessler RC, Adler L, Barkley R, Biederman J, Conners CK, Demler O, et al. The prevalence and correlates of adult ADHD in the United States: results from the National Comorbidity Survey Replication. Am J Psychiatry 2006;163:716-723.

3. Kates N. Attention deficit disorder in adults. Management in primary 
care. Can Fam Physician 2005;51:53-59.

4. Weiss G, Hechtman L, Perlman T, Hopkins J, Wener A. Hyperactives as young adults: a controlled prospective ten-year follow-up of 75 children. Arch Gen Psychiatry 1979;36:675-681.

5. Secnik K, Swensen A, Lage MJ. Comorbidities and costs of adult patients diagnosed with attention-deficit hyperactivity disorder. Pharmacoeconomics 2005;23:93-102.

6. Clarke S, Heussler H, Kohn MR. Attention deficit disorder: not just for children. Intern Med J 2005;35:721-725.

7. Searight HR, Burke JM, Rottnek F. Adult ADHD: evaluation and treatment in family medicine. Am Fam Physician 2000;62:2077-2086, 20912072.

8. Conners C, Erhardt D, Sparrow E. Conners Adult ADHD Rating Scales, Technical Manual. New York: Multi-Health Systems; 1999.

9. Brown T, Gammon G. The Brown Attention Activation Disorder Scale: Protocol for Clinical Use. New Haven (CT): Yale University; 1991.

10. Ward MF. The Wender Utah Rating Scale: an aid in the retrospective diagnosis of childhood attention deficit hyperactivity disorder. Am J Psychiatry 1993;1:885-890.

11. Adler LA, Spencer T, Faraone SV, Kessler RC, Howes MJ, Biederman J, et al. Validity of pilot Adult ADHD Self- Report Scale (ASRS) to Rate Adult ADHD symptoms. Ann Clin Psychiatry 2006;18:145-148.

12. Belendiuk KA, Clarke TL, Chronis AM, Raggi VL. Assessing the concordance of measures used to diagnose adult ADHD. J Atten Disord 2007;10:276-287.

13. Kessler RC, Adler L, Ames M, Demler O, Faraone S, Hiripi E, et al. The World Health Organization Adult ADHD Self-Report Scale (ASRS): a short screening scale for use in the general population. Psychol Med 2005;35:245-256.

14. Sheehan DV, Lecrubier Y, Sheehan KH, Amorim P, Janavs J, Weiller E, et al. The Mini-International Neuropsychiatric Interview (MINI): the development and validation of a structured diagnostic psychiatric in- terview for DSM-IV and ICD-10. J Clin Psychiatry 1998;59(Suppl 20): 22-33.

15. Knouse L, Safren S. Adult Attention-Deficit Hyperactivity Disorder. In: Baer L, Blais MA, Editors. Handbook of Clinical Rating Scales and Assessment in Psychiatry and Mental Health. New York: Humana Press, 2010, p.195-208.

16. Kim JH, Lee EH, Joung YS. The WHO Adult ADHD self-report scale: reliability and validity of the Korean version. Psychiatry Investig 2013;10: 41-46.

17. DuPaul GJ, Power TJ, Anastopoulos AD, Reid R. ADHD Rating ScaleIV: Checklists, Norms, and Clinical Interpretation. New York: Guilford Press; 1998.

18. Kessler RC, Adler LA, Gruber MJ, Sarawate CA, Spencer T, Van Brunt DL. Validity of the World Health Organization Adult ADHD Self-Report Scale (ASRS) Screener in a representative sample of health plan members. Int J Methods Psychiatr Res 2007;16:52-65.

19. Zohar AH, Konfortes H. Diagnosing ADHD in israeli adults: the psychometric properties of the adult ADHD self report scale (ASRS) in hebrew. Isr J Psychiatry Relat Sci 2010;47:308-315.

20. McIntosh D, Kutcher S, Binder C, Levitt A, Fallu A, Rosenbluth M. Adult ADHD and comorbid depression: a consensus-derived diagnostic algorithm for ADHD. Neuropsychiatr Dis Treat 2009;5:137-150.

21. Asherson P, Manor I, Huss M. Attention-deficit/hyperactivity disorder in adults: update on clinical presentation and care. Neuropsychiatry 2014;4:109-128.

22. Faraone SV, Biederman J, Spencer T, Mick E, Murray K, Petty C, et al. Diagnosing adult attention deficit hyperactivity disorder: are late onset and subthreshold diagnoses valid? Am J Psychiatry 2006;163:1720-1729.

23. Mannuzza S, Klein RG, Klein DF, Bessler A, Shrout P. Accuracy of adult recall of childhood attention deficit hyperactivity disorder. Am J Psychiatry 2002;159:1882-1888. 